\title{
Control of Flow Structure on a Semi-Circular Planform Wing
}

\author{
D. Williams ${ }^{*}$, J. Collins ${ }^{\dagger}$, C. Jankhot ${ }^{\ddagger}$ \\ Illinois Institute of Technology, Chicago, IL \\ T. Colonius, \\ California Institute of Technology, Pasadena, CA \\ Gilead Tadmor \\ Northeastern University, Boston, MA
}

\begin{abstract}
Active flow control is used to modify the lift, drag and pitching moments on a semicircular wing with aspect ratio, $A R=2$, and chord Reynolds number is 68,000 . The wing is mounted on a pitch/plunge sting mechanism that responds to the instantaneous loads and moments acting on the wing. The leading edge of the airfoil contains 16 spatially localized actuators that can be independently controlled. Smoke wire visualization, surface pressure and six-component force balance measurements are used to characterize the effects of openloop forcing. The lift coefficients on the steady wing are enhanced with the actuation, similar to the effect of dynamic stall vortex lift enhancement that occurs during a pitch up maneuver. Surface pressure measurements are being used to construct a flow model for use in feedback control. Progress toward the goal of designing a feedback controller to stabilize the flight of the model in an oscillatory freestream is discussed.
\end{abstract}

\section{Nomenclature}

$\begin{array}{lll}\mathrm{b} & = & \text { span at base of model } \\ \mathrm{c} & = & \text { chord on model centerline } \\ \mathrm{C}_{1} & = & \text { sectional lift coefficient } \\ \mathrm{C}_{\mathrm{L}} & = & \text { wing lift coefficient } \\ C_{p} & = & \text { pressure coefficient } \\ \mathrm{C}_{\mu} & = & \text { momentum coefficient for actuator } \\ \mathrm{D} . \mathrm{C} . & = & \text { actuator duty cycle } \\ \mathrm{q} & = & \text { dynamic pressure }=1 / 2 \rho \mathrm{U}^{2} \\ \mathrm{Re}_{\mathrm{c}} & = & \text { Reynolds number based on chord } \\ \mathrm{S} & = & \text { planform area of model }=1 / 2 \pi \mathrm{b}^{2} / 4 \\ \mathrm{U} & = & \text { freestream speed } \\ \alpha & = & \text { angle of attack } \\ \alpha & = & \text { pitch rate }\end{array}$

\section{Introduction}

The overall objective of this experiment is to use pulsed-blowing actuators placed along the leading edge of a wing to modify and control the development of the leading-edge vortex and tip vortex (LEV/TV) system. We aim to extend the range of aspect ratios and Reynolds numbers for which steady lift can be maintained at very high angles of attack $(\alpha)$, while realizing benefits associated with higher aspect ratio aerodynamics during cruise. Control of the leading edge vortex will enhance wing maneuverability by stabilizing or synchronizing vortex

\footnotetext{
${ }^{*}$ Professor, MMAE Dept., Illinois Institute of Technology, 3110 S. State St., Chicago IL, AIAA Associate Fellow.

${ }^{\dagger}$ Graduate Research Assistant, MMAE Dept., Illinois Institute of Technology, 3110 S. State St., Chicago IL.

"Undergraduate Research Assistant, MMAE Dept., Illinois Institute of Technology, 3110 S. State St., Chicago IL.
} 
shedding during pitch, yaw, roll, and in response to wind gusts; and may reduce or eliminate the need for control surfaces made redundant by the flowcontrol actuators.

The premise for this work is based on biological fliers ${ }^{1}$. Insect wings are remarkable for their stable LEV that prevents stall at high angles of attack, but that stability is limited to an aspect ratio less than $\mathrm{AR}=3$ and Reynolds number $(R e) \leq 10^{4}$. There is a strong interplay between the tip vortex and leading edge vorticity generation, and in all flapping flight, vortex shedding is carefully synchronized to regulate the aerodynamic forces and moments. Similarly, previous work in openloop separation control at high $R e$ shows that pulsed air injection can lead to more organized shedding that results in higher lift and smaller mean separation bubbles ${ }^{2}$. However, open-loop performance deteriorates subject to disturbances that desynchronize the actuation-shedding phase lock. To robustly exploit these high-lift mechanisms, we use surface pressure sensors to track the estimated circulation and position of LEV, and apply control based on low-order models ${ }^{3}$.

Our efforts at this stage of the program are focused on constructing a closed-loop control system for regulating the development of the leading edge vortex in unsteady flow conditions. Pressure sensors on the suction surface of the airfoil are used to detect the early stages of stall, which are coincident with the formation of the leading edge vortex. For the first attempt at feedback control for this problem, we are using a quasi-static approach to closed-loop control to adjust the strength of the leading edge vortex in response to an oscillating free stream. A second order differential equation is used to model the response of the airfoil force and moment coefficients.

The experimental setup is described in the next section. Open-loop forcing experiments to correlate lift with the duty cycle of the actuator are discussed in Section III. The results of the closed-loop flight control experiments are described in Section IV, and a summary of the current progress is presented in Section V.

\section{Experimental Setup}

The leading edge and tip vortex interaction studies are being conducted under both steady and dynamic conditions in the Andrew Fejer Unsteady Flow Wind Tunnel shown in figure 1. The chord Reynolds number for the data presented is $\mathrm{Re}_{\mathrm{c}}=68,000$, although it can be varied from $\mathrm{Re}_{\mathrm{c}}=30,000$ to $1,000,000$. A computer controlled shutter at the downstream end of the test section allows the freestream speed to be modulated at frequencies up to 2 $\mathrm{Hz}$. The wing model is mounted on a two-component vertical sting, controlled by Xenus servotubes. The height, pitch angle and pitch rate of the model within the test section are also computer controlled. With this system it is possible to simulate complex flight maneuvers and study the flight response of the wing in an unsteady flow environment.

A cutaway view of one half span of the airfoil model is shown in figure 2. The planform is a semi-circle with a centerline chord $\mathrm{c}=203 \mathrm{~mm}$, and span $\mathrm{b}=406 \mathrm{~mm}$ and aspect ratio $=2.54$. Steady spanwise directed blowing is known to stabilize the leading edge vortex on rectangular wings, but the mass flow rate requirements were quite $\operatorname{large}^{4}$. In this experiment we use unsteady, pulsed-blowing with 16 micro-valves actuation that are located

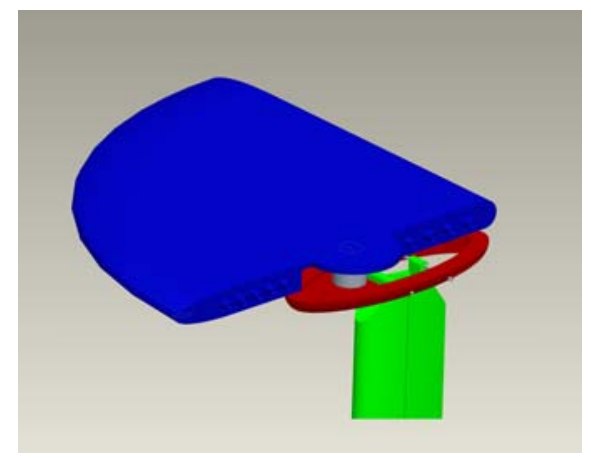

Figure 2 - Right half of the 3D airfoil model with aspect ratio $=2$. 
along the leading edge. Each actuator is isolated from its neighbor, and can be individually activated to produce traveling wave patterns. However, at this stage in the project we are driving all actuators in phase. To document the open-loop forcing effects on performance, the actuators are operating at $25 \mathrm{~Hz}$ pulse rate, and a $\mathrm{C}_{\mu}^{\S}=0.0074$.

The transient response of the leading edge vortex and the tip vortex system to open-loop forcing by the actuators, such as, impulse and step inputs, was obtained for modeling and validation purposes. The influence of the vortex system on the airfoil lift, drag and moment coefficients is computed from surface pressure distributions using two surface pressure taps at $\mathrm{x} / \mathrm{c}=0.42$ and $\mathrm{x} / \mathrm{c}=0.72$, and an ATI force 6-component balance system.

\section{Results from Open Loop Forcing Tests}

Smoke wire flow visualization of the semi-circular airfoil at $19^{\circ}$ angle of attack is shown in figure $3 \mathrm{a}$, $\mathrm{b}$. The smoke sheet is positioned at a center chord. As expected, without flow control the flow is fully separated at this angle of attack, figure 3a. Activation of the 16 pulsed-blowing actuators (all in phase with each other) along the leading edge reattaches the flow as shown in figure $3 \mathrm{~b}$. It will be shown later that the maximum lift coefficient is increased from $\mathrm{C}_{\mathrm{L}}=0.99$ to 1.6 with this type of open-loop actuation.

When the smoke sheet is repositioned near the quarter-span of the airfoil as shown in figure $4 \mathrm{a}, \mathrm{b}$, the fully separated region is reduced to a smaller separation bubble near the leading edge. There is a possibility that the leading edge vortex has been stabilized in this case, i.e., the small separated region is actually a concentrated vortex. However, more detailed measurements with our PIV system of the circulation are needed to document the strength of the leading edge vortex.

It is well known that airfoils and wings pitched upward at high pitch rates form dynamic stall vortices. The extra circulation associated with these vortices leads to higher lift coefficients than can be achieved under steady
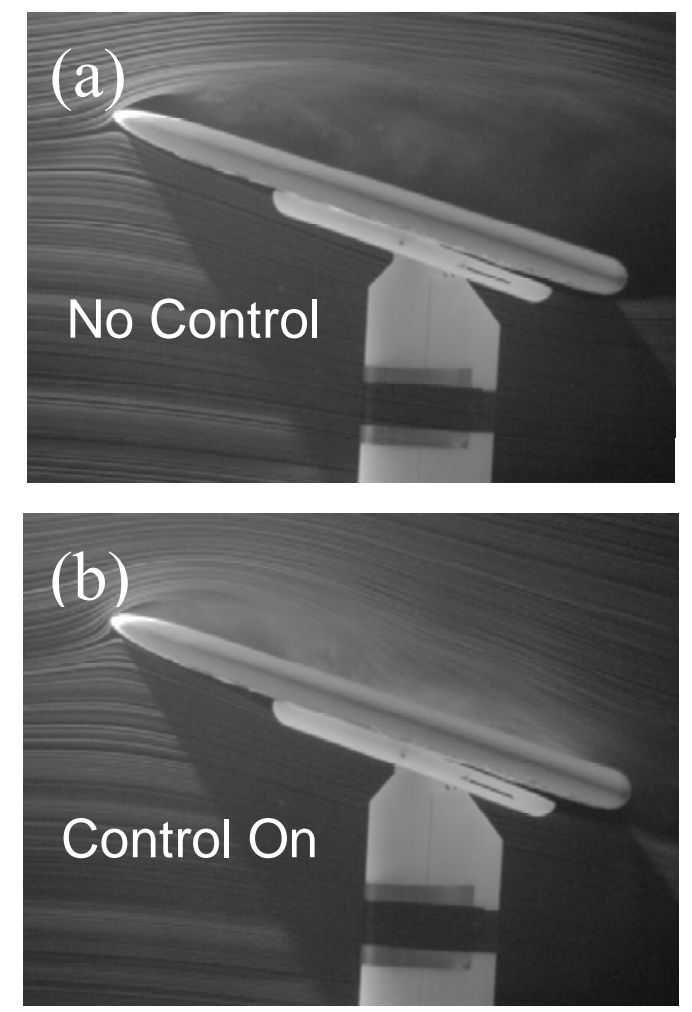

Figure 3 - Smoke wire visualization of the flow at the center-span region of the wing. a) no forcing, $\alpha=19^{\circ}$; b) pulsed-jet actuation, $\alpha=19^{\circ}, \operatorname{Re}_{\mathrm{c}}=68,000$.
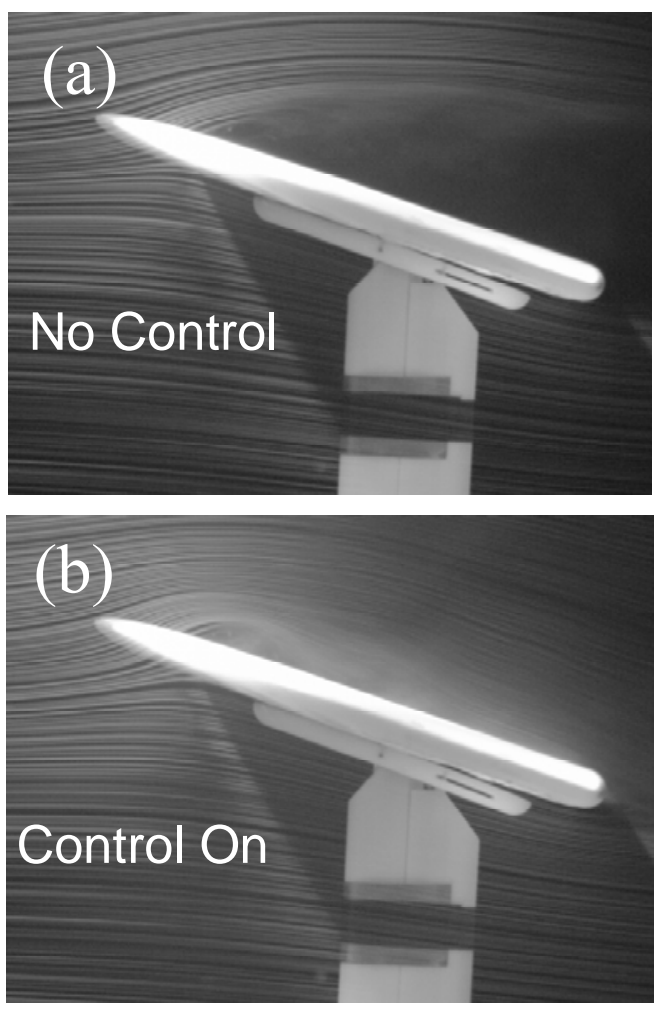

Figure 4 - Smoke wire visualization of the flow near the quarter span of the semicircular wing. a) no forcing, $\alpha=19^{\circ}$; b) pulsed-jet actuation, $\alpha=19^{\circ}, \operatorname{Re}_{\mathrm{c}}=68,000$.

\footnotetext{
${ }^{\S} \mathrm{C}_{\mu}$ values are based on the time averaged mass flow rate to all 16 micro valve actuators.
} 
conditions. The lift coefficient results in figure 5 indicate that our semi-circular wing exhibits the same characteristics. Steady behavior is achieved by using a slow pitch rate of $\alpha^{\prime}=0.9 \mathrm{deg} / \mathrm{sec}$. Stall under near static conditions occurs at $\alpha=16^{\circ}$ and a maximum $C_{L}=0.99$ is obtained. The higher pitch rates of $\alpha^{\prime}=40$ and $80 \mathrm{deg} / \mathrm{sec}$

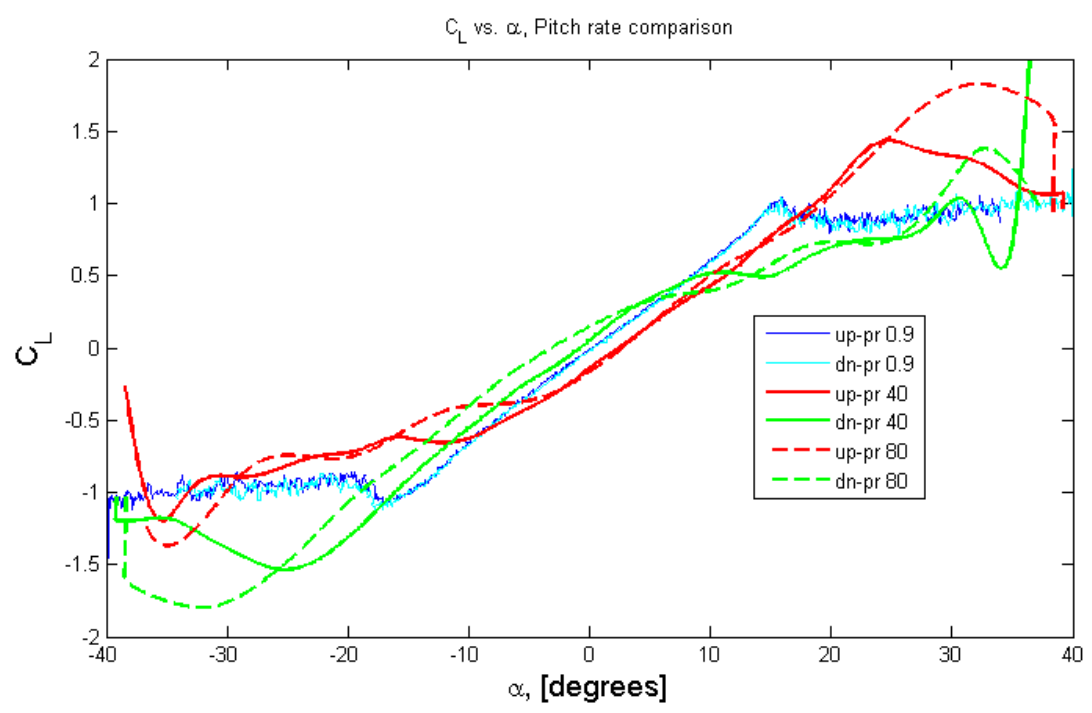

Figure 5 - Lift, drag and pitch moment coefficients. No forcing at pitch rates $\alpha^{\prime}=0.9,40$, and $80 \mathrm{deg} / \mathrm{sec}$.

delay stall to $\alpha=24^{\circ}$ and $32^{\circ}$, respectively. The presence of a dynamic stall vortex increases the lift coefficients to $C_{L}$ $=1.4$ and $\mathrm{C}_{\mathrm{L}}=1.8$ for the two pitch rates. Pitch-down maneuvers at the same constant rates are also shown in the figures to demonstrate the symmetry of the result. Since the wing has no camber, the symmetry was expected.

The effect of open-loop forcing on the wing lift coefficient is shown in figure 6. For reference purposes, the pitch up and pitch down baseline cases with actuators turned off are shown by the curves labeled " $\mathrm{f}=0$." The actuators are operated at $25 \mathrm{~Hz}, \mathrm{C}_{\mu}=.0074$, and pitch rates were $\alpha^{\prime}=0.9$ and $40 \mathrm{deg} / \mathrm{sec}$. The effect of actuation on the "steady" lift coefficient is shown by the thick blue line. Stall is delayed until $\alpha=23^{\circ}$ where a maximum lift coefficient of $\mathrm{C}_{\mathrm{L}}=1.6$ is reached. The heavy solid red line shows the lift coefficient at a pitch rate of 40 $\mathrm{deg} / \mathrm{sec}$ with the actuators turned off. It is quite interesting that the steady-state response to forcing is similar to the dynamic stall vortex behavior in that both produce the same delay in stall angle and extra lift. This is indirect evidence supporting our earlier conjecture that the actuation is stabilizing the leading edge vortex.

Since closed-loop control will be used to obtain the same high lift coefficient values during wing maneuvers and flight in an unsteady freestream, knowledge of the flow state

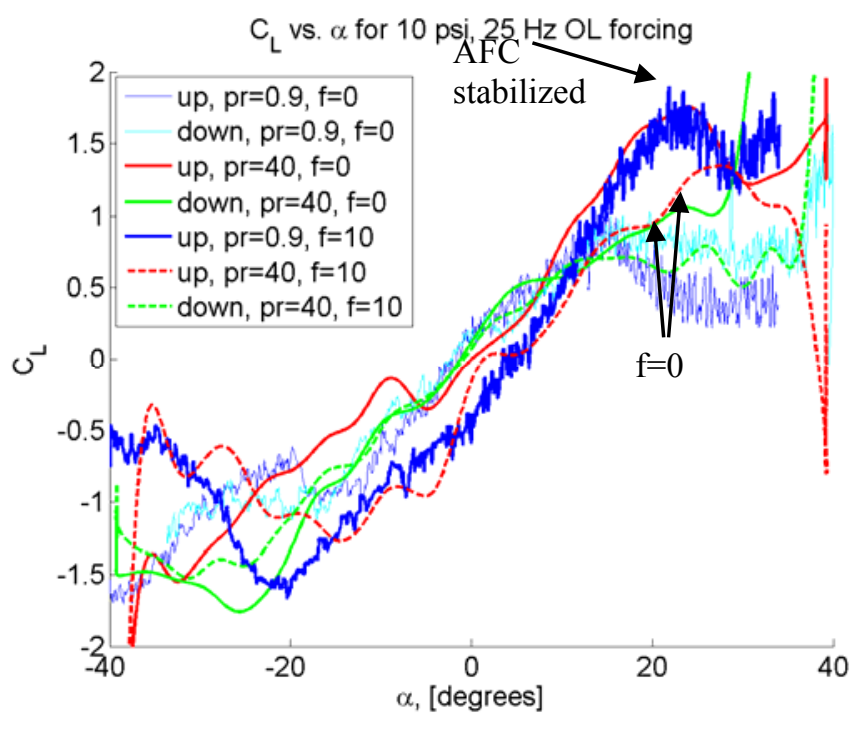

Figure 6 - Lift, drag and pitch moment coefficients. Forcing on at $25 \mathrm{~Hz}, \mathrm{C}_{\mu}=.0074$ and pitch rates $\alpha^{\prime}=0.9$ and $40 \mathrm{deg} / \mathrm{sec}$. Forcing in the "static" case produces same lift coefficient as the dynamic case.

on the wing is an essential element to the development of the controller. We are using the lift force and pressure taps located at $\mathrm{x} / \mathrm{c}=0.42$ and 0.72 as feedback and flow state sensors, respectively. Figures 7 and 8 show the mean 
pressures measured at $\mathrm{x} / \mathrm{c}=0.42$, corresponding to $\alpha^{\prime}=0.9$ and $40 \mathrm{deg} / \mathrm{sec}$ pitch-up and pitch-down maneuvers. The quasi-steady data at $\alpha^{\prime}=0.9$ is used as input to a second order differential equation model, which predicts the effects of the pitch rate on the pressure. Figure 7 shows that the pressure decreases linearly prior to flow separation, irrespective of the pitch rate. During a pitch up maneuver, the separation is delayed (red dashed line), and during pitch down the reattachment of the flow is delayed (dash-dot line).

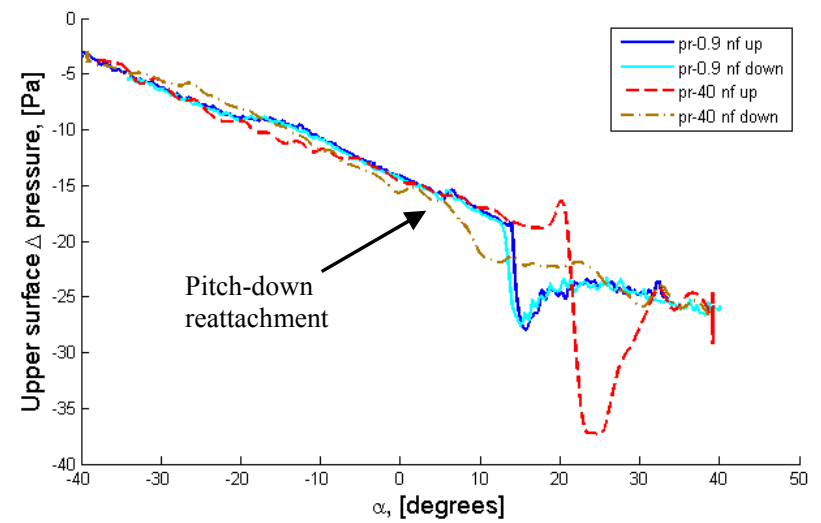

Figure 7 - Surface pressure measured at $\mathrm{x} / \mathrm{c}=.42$. No forcing at wing pitch rates $\alpha^{\prime}=0.9$ and 40 deg/sec.

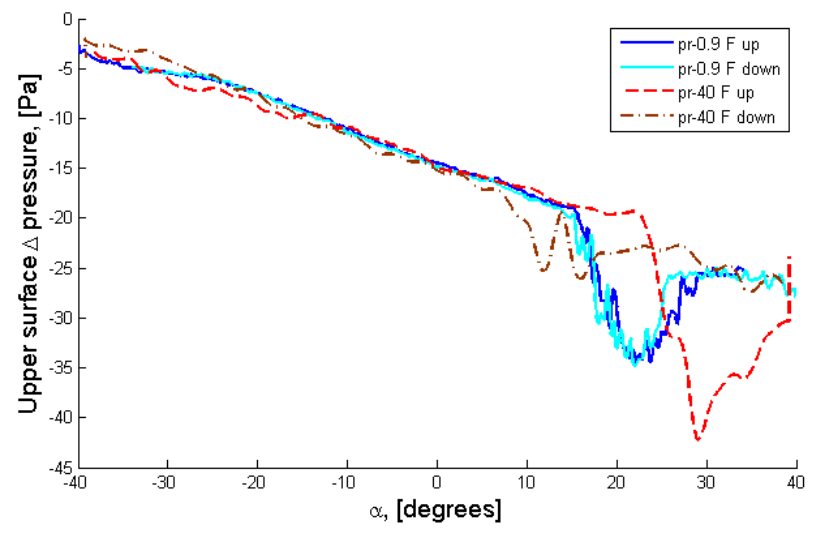

Figure 8 - Surface pressure measured at $\mathrm{x} / \mathrm{c}=.42$. Forcing on at $25 \mathrm{~Hz}, \mathrm{C}_{\mu}=.0074$ and pitch rates $\alpha^{\prime}=0.9$ and $40 \mathrm{deg} / \mathrm{sec}$.

The effect of actuator forcing on the surface pressure is shown in figure 8. The pressure response to actuation is somewhat different from the response to pitch rate shown in figure 7. Consequently, it will be necessary to include the effect of actuation in the flow state model in order to have an effective closed-loop control system. We are currently determining the model coefficients necessary to include these effects. The following section discusses the overall control architecture.

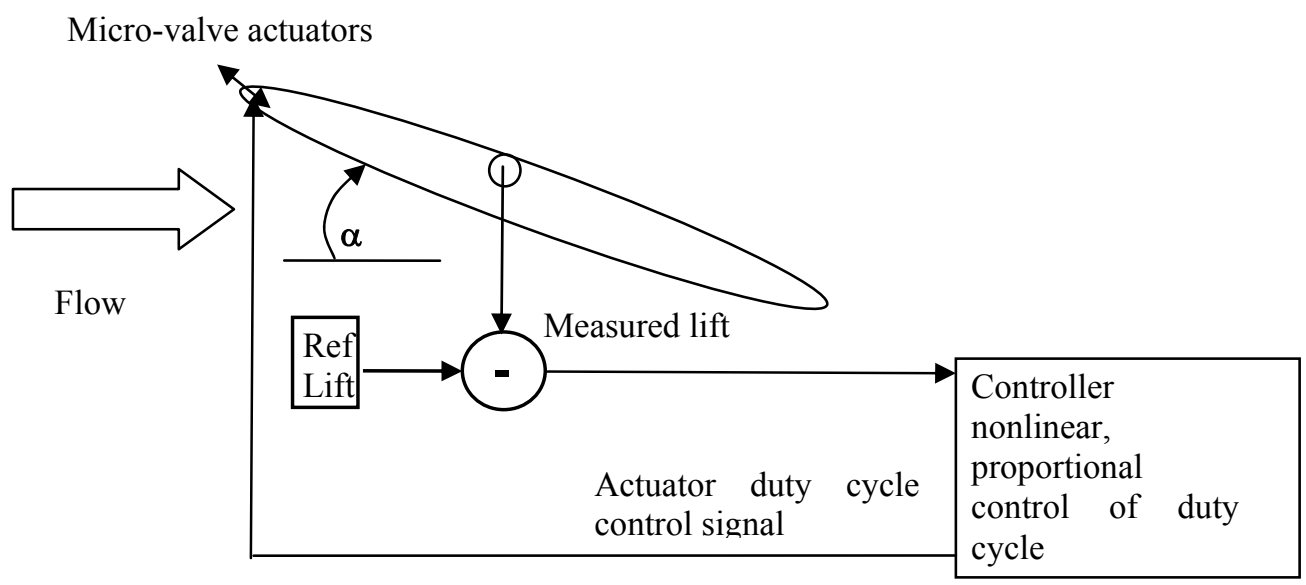

Figure 9 - Schematic of control system architecture.

\section{Closed Loop Lift Control}

As a first attempt at closed loop control in an unsteady flow, the freestream speed in the test section of the wind tunnel was oscillated sinusoidally about a mean value with the wing was fixed at $\alpha=19^{\circ}$ angle of attack. At $19^{\circ}$ angle of attack the wing is in the post-stall region where the actuation was most effective in modifying the lift. The mean speed of the freestream was $5.0 \mathrm{~m} / \mathrm{s}$ with oscillation amplitude of $+/-0.5 \mathrm{~m} / \mathrm{s}$. The control objective is to 
maintain a constant lift force in the oscillating freestream flow using the simple proportional control architecture shown in figure 9. Ultimately, a more sophisticated approach to control, such as, using an observer similar to Magill, et al. ${ }^{5}$ will be designed, but at this early stage we want to keep the approach as simple as possible.

The open-loop forcing experiments showed that the most effective forcing frequency was $25 \mathrm{~Hz}$ pulse rate for the actuators. The duty cycle (D.C.) of the actuator pulse train can be changed more rapidly than the supply pressure leading to the micro-valves, which gives a higher bandwidth actuator response. The change in lift force and pitching moment that occurs with changing duty cycle is shown in figure 10. D.C. $=0$ corresponds to valves in a continuously closed state, and D.C. $=100$ percent corresponds to continuous pulsing of the valves at $25 \mathrm{~Hz}$.

Lift force transient response experiments were conducted that determined the flow field required approximately 0.3 seconds to respond to a step change in input. As a starting point, it was decided to use 7 cycles of the $25 \mathrm{~Hz}$ pulse train as the minimum time unit of actuation. The duty cycle was then defined as the number of pulses out of the 7 that the micro-valves are open. As shown in figure 10 , with D.C. $=0$ the valves are continuously closed and the lift is $0.77 \mathrm{~N}$ at $\alpha=19^{\circ}$. Increasing the D.C. to 100 percent leaves the micro-valves running continuously at $25 \mathrm{~Hz}$ and increases the lift to $1.2 \mathrm{~N}$.

The effect of freestream oscillation on the lift force is shown in figure 11 as the dashed red line. The 10 cycles of lift oscillation and the first harmonic are clearly visible in the 20 seconds of data. The solid green line represents the lift force produced by the actuator running in open-loop, $0.5 \mathrm{~Hz}$ sinusoidal duty cycle variation. Again, a significant amount of harmonics is produced by the actuator. Nevertheless, the actuator appears to have sufficient authority, because it produces comparable amplitudes to the lift fluctuation produced by the oscillating freestream.

The next step in the process is to close the loop, so that the lift oscillations produced by the actuator will offset the lift fluctuations from the freestream variation. From figure 10 we see a nonlinear relationship between the lift increment and the duty cycle. For nonlinear proportional control, we used the expression D.C. $=\left(\left((\Delta L i f t+0.3)^{2} \times 5.5\right)\right.$ to obtain the required fractional duty cycle value, where $\Delta \mathrm{Lift}$ is the commanded lift increment value. A $\Delta \mathrm{Lift}$ value of -0.3 produces $\mathrm{DC}=0$, and a $\Delta \mathrm{Lift}=.13$ produces $\mathrm{DC}=1$.

With the elements of a simple proportional feedback controller nearly complete, we will begin our attempts to maintain a constant value of lift in an unsteady flow environment.
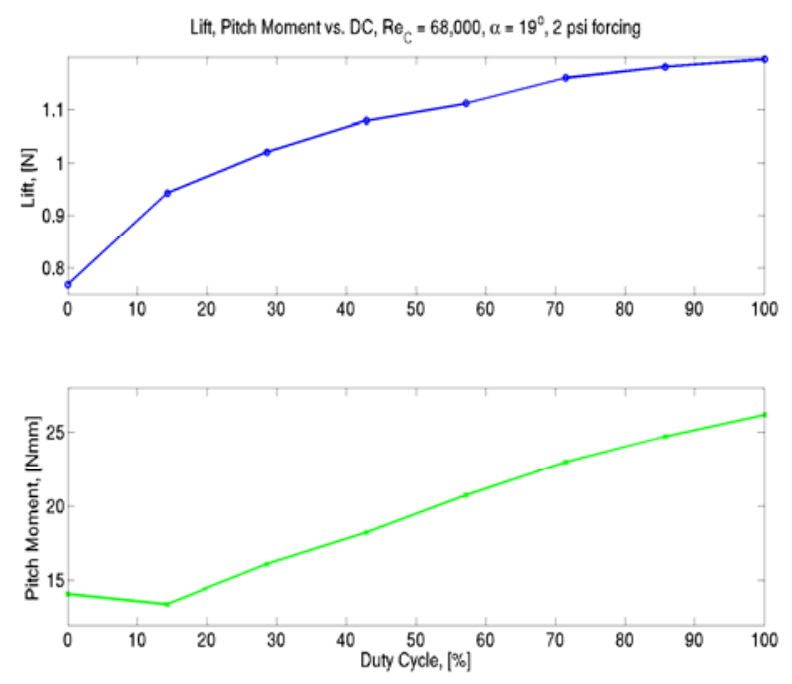

Figure 10 - Lift and pitching moment dependence on actuator duty cycle. Micro-valve frequency is $25 \mathrm{~Hz}$, with supply pressure of 2 psig, and $C_{\mu}=.0074$.

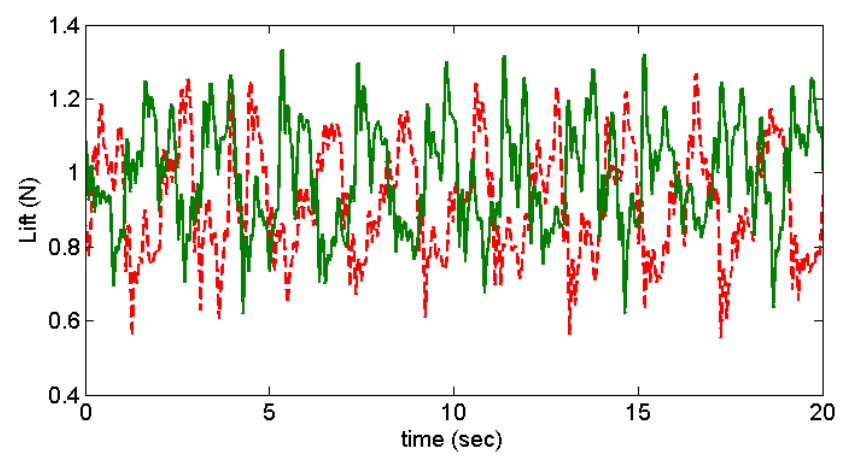

Figure 11 - Lift produced by oscillating freestream dashed, red line; Lift produced by sinusoidally varying actuator - green, solid line. 


\section{Conclusions}

Wind tunnel experiments confirmed that the flow around a semi-circular wing can be modified using 16 microvalve actuators placed along the leading edge. The effect of the actuation is to produce extra lift by stabilizing a region of concentrated vorticity near the leading edge of the wing. The extra lift and the delay of stall associated with the concentrated vorticity results in a lift-curve slope similar to the one produced by a dynamic stall vortex. Flow visualization provided clear indication of the presence of the stabilized leading edge vortex.

Measurements of the surface pressure at two locations along the centerline of the airfoil $(\mathrm{x} / \mathrm{C}=0.42$ and 0.72$)$ also support the similarity between the controlled leading edge vortex and the dynamic stall vortex. By correlating the surface pressure signals with the overall lift coefficient and pitching moment, we expect to be able to determine the instantaneous flow state for use in closed-loop control.

A simple closed-loop control system is being developed to maintain constant lift in a wind tunnel with an oscillating freestream flow. To achieve good time response (bandwidth) from the micro-valve actuator system, a quantized, variable duty cycle approach is used. Modulation of the lift by changing the duty cycle of actuation was demonstrated in the post-stall region of the wing. The control authority in changing the lift force with actuation is comparable in amplitude to the lift oscillations produced by the sinusoidally varying freestream, which gives confidence that it will be possible to maintain a steady value of lift in an unsteady freestream.

\section{Acknowledgement}

The support for this work by the U.S. Air Force Office of Scientific Research MURI (FA9550-05-1-0369) with program manager Dr. Fariba Fahroo is gratefully acknowledged. We are also appreciative of the support for Jesse Collins from the Illinois NASA Space Grant Consortium.

\section{References}

1. Birch, J.M., Dickson, W.B., Dickinson, M.H., "Force production and flow structure of the leading edge vortex on flapping wings at high and low Reynolds numbers," J. of Experimental Biology, vol. 207, pp. 1063-1072.

2. Williams, D.R., Doshi, S., Collins, J., Colonius, T., "Control of the Spanwise Distribution of Circulation on NACA 0012 and Flat Plate Wings," AIAA Paper 2007-1121.

3. Colonius, T., Rowley, C.W., Tadmor, G., Williams, D.R., Taira, K., "Closed-loop Control of leading-edge and tip vortices for small UAV," $1{ }^{\text {st }}$ Conference on Active Flow Control, Berlin Germany, Sept. 27-29, 2006.

4. Dixon, C.J., "Lift Augmentation by Lateral Blowing Over a Lifting Surface," AIAA Paper 69-193, AIAA/AHS VTOL Research, Design and Operations Meeting, Atlanta, GA, Feb. 1969.

5. Magill, J.C., Buchmann, M., Rixon, G., and McManus, K., "Dynamic Stall Control Using a Model-Based Observer," J. Aircraft, Vol. 40, No. 2, 2003, pp. 355-362. 